\title{
Integration of Physician and Nursing Professional Efforts to Deliver Supportive Scalp Cooling Care to Oncology Patients at Risk for Alopecia
}

Lindsay L. Peterson - Maryam Lustberg - Sara M. Tolaney •

Mikel Ross · Elahe Salehi · Steven J. Isakoff

Received: May 15, 2020 / Published online: June 19, 2020

(C) The Author(s) 2020

\section{ABSTRACT}

Scalp cooling (SC) is an effective and generally well-tolerated method for prevention of chemotherapy-induced alopecia (CIA). Initially studied in early-stage breast cancer, these devices have expanded US Food and Drug Administration (FDA) clearance in a broad range of solid tumors including ovarian, colorectal, and prostate. Introducing SC to eligible patients, including those distraught by concerns of CIA, requires an integrated effort on the part of the physician, nursing, and care manager medical team. This article presents a pragmatic workflow

Digital Features To view digital features for this article go to https://doi.org/10.6084/m9.figshare.12453296.

L. L. Peterson $(\bowtie)$

Division of Medical Oncology, Washington

University School of Medicine, St. Louis, MO, USA

e-mail: llpeterson@wustl.edu

M. Lustberg

Comprehensive Cancer Center, The Ohio State

University, Columbus, OH 43210, USA

S. M. Tolaney · E. Salehi

Dana Farber Cancer Institute, Boston, MA, USA

M. Ross

Memorial Sloan Kettering Cancer Center, New York, NY, USA

S. J. Isakoff

Massachusetts General Hosp Cancer Center, Boston, MA, USA for collaborative efforts from physicians and allied health professionals in the USA to deliver supportive SC to reduce CIA in patients undergoing treatment regimens known to impact hair follicles. It further highlights the efforts required to identify appropriate patients, educate, and set expectations of patients. The supervisory role of the physician during the procedure, the nursing role in monitoring and documentation, and the post-procedure decision-making by the physician are also addressed. Lastly, it suggests that integrated physician and nursing efforts necessary for scalp cooling are similar to other care used in oncology.

Keywords: Adverse effects; Alopecia; Chemotherapy; Cold caps; Quality of life; Scalp cooling; Solid tumors; Supportive care 


\section{Key Summary Points}

Scalp cooling (SC) to prevent or minimize chemotherapy-induced alopecia (CIA) has received expanded Food and Drug Administration (FDA) approval for patients with most solid tumors.

The National Comprehensive Cancer Network (NCCN) Clinical Practice Guidelines in Oncology (NCCN Guidelines ${ }^{\circledR}$ ) now recommend SC as a category 2A treatment option for patients with invasive breast cancer and ovarian cancer, fallopian tube cancer, and peritoneal cancer.

The integrated roles of physicians, advanced practitioners, and nurses to implement supportive care with SC, including the steps of identifying appropriate patients for SC, educating patients about the procedure, setting expectations for outcomes, placing orders, and overseeing, monitoring, and documenting results, have been delineated.

Physician, advanced practitioner, and nursing responsibilities for SC interventions are similar to current functions delivering supportive care for other adverse events associated with cancer treatment such as nausea and vomiting, pain, anemia, and fatigue.

\section{INTRODUCTION}

Several cytotoxic drugs are known to cause moderate to severe alopecia [1]. Chemotherapyinduced alopecia (CIA) usually occurs 7-14 days after infusion. The incidence and severity of alopecia depend both on the type and dose of chemotherapy [1]. CIA is a distressing adverse event associated with the use of systemic chemotherapy in patients with cancer. Hair loss impacts self-esteem, is a visible reminder of the disease, affects the patient's privacy, and negatively impacts social and work interactions $[2,3]$. In a patient survey, the impact of CIA on patients ranks highest, ahead of fatigue, nausea, trouble sleeping, and early hot flashes [3].

The theory as to why inducing scalp hypothermia limits CIA is twofold. First, cooling constricts blood vessels in the scalp, thereby decreasing the amount of chemotherapy that reaches the hair follicles. Second, the cold decreases the proliferative activity of hair follicles and makes them less sensitive to cytotoxic drugs, which target rapidly dividing cells. Consequently, there is less of a chemotherapeutic effect on hair follicle cells which limits hair loss from the scalp, without impacting overall treatment efficacy [4].

Scalp cooling (SC) devices are US Food and Drug Administration (FDA)-cleared and indicated to reduce the likelihood of CIA in patients with solid tumors such as ovarian, breast, colorectal, bowel, and prostate cancer [5]. These systems allow the scalp to be cooled by circulating cold fluid through channels in the inner cap specifically fitted to the patient. The temperature of the coolant is monitored and kept within a specified temperature range $\left(23-25^{\circ} \mathrm{F}\right)$.

SC efficacy is dependent on several factors, the most significant being the chemotherapy regimen (drugs, dose, frequency, number of cycles). While individual factors such as hair type can play a role in efficacy, the extent to which these factors affect outcome remains anecdotal. The National Comprehensive Cancer Network (NCCN) Guidelines ${ }^{\circledR}$ recommend SC as a category $2 \mathrm{~A}$ treatment option for patients with invasive breast cancer and ovarian cancer $[6,7]$.

Efficacy of SC is based on a visual inspection of the patient's scalp by a clinician during physical exam, or on a patient's alopecia selfreport. SC significantly reduces the risk of CIA from taxane-based and anthracycline-based chemotherapy regimens [8-12]. The majority of studies have been conducted in patients with early-stage breast cancer receiving neoadjuvant or adjuvant chemotherapy with curative intent $[13,14]$. However, one prospective trial and one large registry analysis performed in patients 
with breast, lung, prostate, gastrointestinal/colorectal, and female genital solid tumors demonstrated the potential for broader use, including in the palliative setting $[15,16]$.

SC has minimal adverse events, is a generally well-tolerated intervention, and contributes to patient well-being $[3,17]$. The most common SC-related adverse events are grade 1 and include chills, "heavy head", headache, pain (scalp, forehead, or sinus), nausea, dizziness, pruritus, and skin ulceration [9, 12, 13]. No serious adverse events have been reported from the device and participants find the SC device to be reasonably comfortable [9]. In general, very few patients discontinue SC because of intolerance or side effects from the intervention [11, 14-16].

Initially there was concern that SC could increase the risk of scalp metastases. This concern created a barrier to incorporation of the technology into standard cancer care; however, subsequent studies did not show this to be a significant risk. In a study of Dutch patients with cancer and 5 years of follow-up, no scalp metastases occurred in patients receiving the SC intervention. A review of 58 publications including 533 women with breast cancer reported that only $0.36 \%$ of patients receiving SC developed scalp metastases [14] and that overall mortality was not affected in women who received SC (HR 0.89, 95\% CI 0.68-1.17, $p=0.40)$ [18]. Another meta-analysis including 1959 patients reported that the incidence rate of scalp metastasis in the scalp cooling group versus the no scalp cooling group was $0.61 \%(95 \%$ CI $\quad 0.32-1.1 \%)$ versus $0.41 \% \quad(95 \% \quad$ CI $0.13-0.94 \%) ; p=0.43$ [19]. Therefore, the fear of increased risk of scalp metastases has not been demonstrated in research or practice and should not limit the use of SC technology.

SC technology has been around for over a quarter of a century. At this time, the Paxman System and the DigniCap Scalp Cooling System have been cleared by the FDA and are marketing in the USA. The Paxman SC system operates in over 1156 locations and has helped over 100,000 patients with cancer worldwide in over 50 countries to retain their hair during chemotherapy $[20,21]$. Study results have demonstrated a high degree of patient satisfaction using SC and that a majority of patients reported not needing to wear a head covering during the time course of chemotherapy $[8,13,14]$. Dignitana markets an SC system called the DigniCap that is available in 39 countries. A second system called DigniCap Delta received clearance from the FDA in June 2019 and CE Marking in March 2019. More than 120 DigniCap Deltas are operating in the USA and around the globe [22].

Using a SC system will extend the patient's time at the treatment center or clinic. SC starts at least $30 \mathrm{~min}$ before the infusion of cytotoxic chemotherapy. Cooling continues throughout the infusion of chemotherapeutic agents and for up to $20-120 \mathrm{~min}$ after the infusion is completed, dependent on the chemotherapy regimen $[14,23]$. Treatment centers must incorporate the logistics of SC into facility workflows by coordinating physician general supervision of the patient, as defined by the 2020 Hospital Outpatient Prospective Payment System (HOPPS) final rule [24], scheduling additional chair time, planning for incremental nursing staff effort, and modifying the physical infusion suite to accommodate cooling machines (e.g., space, power requirements) [25].

Physicians play an important role in the decision to offer SC to patients, the education that must be provided to patients, and the general management of SC for CIA before, during, and after delivery of chemotherapy. The hands-on roles of the chemotherapy nurse and nurse manager in the process are also critical. Interaction and proper communication between physicians, advanced practitioners, and allied health professionals are needed to provide this supportive care service to patients at risk for CIA.

\section{DISCUSSION}

The incidence and severity of hair loss depend both on the type of cytotoxic drug and the dose of chemotherapy used [1]. Although CIA is mostly reversible, there are rare cases of permanent CIA [23]. Even when CIA is temporary, for most patients it is still a most distressing side effect of treatment $[2,3]$. It is important for the 


\section{INTEGRATED SCALP COOLING WORKFLOW}
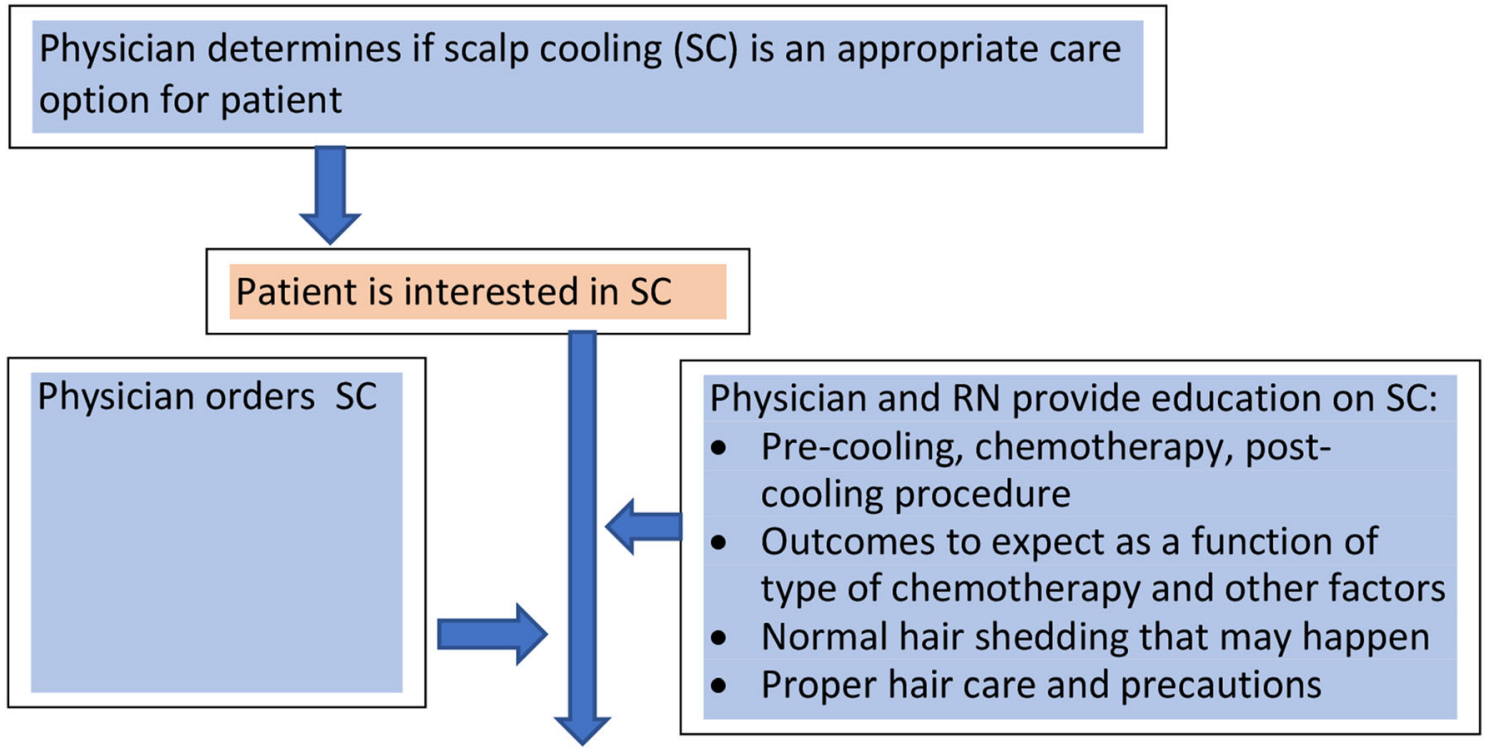

- Scheduler adjusts chemotherapy appointments to include SC

Physician notified if patient is not tolerating SC and requires intervention, or if device malfunction. Physician adjusts treatment for next cycle based on assessment and discussion with nurse.

- Cap sizing completed by RN before first appointment

\section{RN}

- Oversees fitting and proper connection of SC device

- Pre-cooling, cooling during chemotherapy, and post-cooling of patient

- Patient assessed pre-, during, and postchemotherapy

- Documents procedure upon completion

Physician will

- Visually inspect scalp regions of concerns

- Have patient report on their assessment of outcomes

- Adjust the chemotherapy regimen and scalp cooling intervention, as necessary 
4Fig. 1 Integration of physician and nursing staff into the scalp cooling care workflow for treatment of chemotherapy-induced alopecia. RN registered nurse

healthcare team to understand how important the issue of hair loss is to each patient and whether that patient would benefit from an intervention that might minimize hair loss and contribute to an improvement in patient-reported outcomes. Figure 1 provides a workflow that defines the role of physicians, advanced practitioners, and nurses, as they work together to deliver SC to appropriate patients. The first step is providing the necessary education to the patient and their families with respect to the cooling procedure, expected outcomes, normal hair shedding that may occur, and proper hair care and precautions.

For this education to be effective, clinicians need to be aware of the available data on hair preservation and restoration associated with SC [8-12] and be able to counsel patients on the likelihood of success with various chemotherapy regimens. Decision aid websites are available (https://www.scalpcooling.org/ and https://coldcap.com/cold-capping/is-it-for-me/) that allow physicians to enter the type of chemotherapy and dose they plan to use for a particular patient and obtain an estimated success rate of hair retention with the use of SC that they can share with the patient. The number provided is an approximation based on the Dutch Scalp Cooling Registry [10, 12]. While individual results may ultimately be better or worse, this allows physicians to set realistic expectations for their patients. This is important because results from patient questionnaires demonstrate that patients experience additional distress if they experience hair loss despite SC [3].

Proper communication between physicians and patients can help the patient decide whether to consider using the SC intervention. When the physician discusses the cancer treatment plan (choice of regimen, frequency of administration, dose of drug(s), number of dosing cycles, possible therapeutic outcome, and adverse events), they can also share
Table 1 Physician-patient areas of discussion regarding scalp cooling procedure

\begin{tabular}{ll}
\hline $\begin{array}{l}\text { Pros and cons of supportive } \\
\text { care for CIA }\end{array}$ & Clinical benefits \\
& Discomforts \\
Adverse events & Risks \\
Expectations from SC & Outcomes as a function of \\
Treatment regimen & Dose schedule \\
Factors that influence success & Type of chemotherapy \\
with SC & Dose \\
& Ability of patient to \\
tolerate coldness \\
Re- and post-cooling time \\
Thickness of patient's hair \\
Howter with SC
\end{tabular}

$C I A$ chemotherapy-induced alopecia, $S C$ scalp cooling

information and respond to patient queries regarding supportive care for CIA. Table 1 provides a list of topics that patients may want to know more about. These topics include the benefits and risks of SC, anticipated outcomes, factors that might influence outcomes, and amount of experience that the treatment center has with the SC procedure.

Many factors beyond the type of chemotherapy and dose of drugs influence how effective the procedure is at any given treatment center. For example, the ability of patient to tolerate the cold temperature, the amount of pre- and post-cooling time allotted, the thickness and length of the patient's hair (thicker is more insulating), and how well the cap is fitted are some of the key considerations $[1,4,16]$. Anxiety can also reduce tolerability to SC, 
which can reduce effectiveness. Therefore, use of anti-anxiety medication can be useful.

The physician provides general supervision of the procedure and is available by telephone to provide assistance and direction as needed. Together with nurse practitioners (NPs) and physician assistants (PAs), physicians must be aware of these factors and be able to discuss them with patients during a visit or after the patient returns home and has additional concerns. Some institutions have created a "steps in the process" sheet and a "physician's talking points" sheet with a checklist that serves as a guide for patient education and cap sizing [25].

If the patient wishes to pursue SC, the physician must prescribe the SC, similar to orders and prescriptions for chemotherapy. Time must be allotted for cap fitting prior to initiation of treatment. The patient's decision to pursue scalp cooling can have implications for the sequence in which physicians order multiagent chemotherapy. For example, in patients with breast cancer who will receive both an anthracycline (AN) and taxane (T), the typical sequence is anthracycline followed by taxane. However, if the physician reverses the sequence and prescribes the taxane prior to the anthracycline this will increase the likelihood of a longer duration of hair retention, as SC is more effective with taxanes than with anthracyclines. Several papers have reported a higher overall success rate for SC when an anthracycline-containing regimen is delivered in a T-AC fashion $[10,13]$. Additional chair time must be scheduled for the pre- and post-cooling steps. During the full cooling procedure, the patient must be monitored by the nursing team and managed in the event that any clinical issues (e.g., headache, anxiety, discomfort, and poor tolerability of the cold) or technical difficulties with the cooling apparatus arise. The physician's office is typically adjacent to the infusion suites. The physician or other qualified healthcare professional provides general supervision during SC, ensuring their immediate availability to the staff in the office, periodically assessing the patient and the patient's response to treatment, and interruptible to provide assistance and direction throughout the performance of the SC procedure [26]. Documentation is provided for all orders, alerts, and tolerability issues and assessments before and after SC intervention (personal communication). The physician assesses the outcomes of SC on CIA during an interval follow-up visit with the patient.

Implementation of SC to minimize CIA by an integrated physician and nursing team is similar to other supportive care provided in the oncology setting to minimize other chemotherapy-induced toxicities such as nausea and vomiting, pain, febrile neutropenia, thrombocytopenia, anemia, and fatigue. The physician has an active role is anticipating the magnitude of the problem with different treatment regimens, discussing risks with the patient, placing orders for antiemetics, analgesics, colony-stimulating factors, transfusions, and trouble-shooting, and adjusting management based on outcomes from patient monitoring [27]. As such, physicians, nurses, and treatment center managers working in an integrative fashion apply supportive care scenarios to the management of CIA in breast cancer and other solid tumor indications $[15,16]$.

Physicians play an important role in educating, setting expectations, and addressing patient concerns regarding CIA and the value of SC. Physicians are providing general supervision at a minimum and are on the floor and available by phone to provide assistance and direction if a complication or need arises during the precooling, chemotherapy, or post-cooling phases. The ability to provide SC care may be limited by the available facility space, and available nursing time.

\section{CONCLUSIONS}

Supportive care within oncology is largely the responsibility of medical and nursing professionals. Interventions that reduce the impact of adverse events can make a significant difference in how patients deal with their illness over time and short- and long-term quality of life. This is particularly true for scalp cooling, which can minimize chemotherapy-induced alopecia which is often the most distressful side effect of chemotherapy and cancer care. The ability of 
physicians and allied healthcare professionals to work in an integrated manner and ascertain the patient's needs for support, educate the patient on benefits and risks, set appropriate expectations of outcomes, and monitor and manage the effect of supportive interventions can provide valuable care in the management of CIA.

\section{ACKNOWLEDGMENTS}

Funding. Paxman Inc. has provided funding to cover the journal's Rapid Service Fee.

Editorial Assistance. Editorial assistance in the preparation of this article was provided by Dr. Rosalyn Blumenthal of Innovation Partners Inc. Support for this work was funded by Paxman Inc.

Authorship. All named authors meet the International Committee of Medical Journal Editors (ICMJE) criteria for authorship for this article, take responsibility for the integrity of the work as a whole, and have given their approval for this version to be published.

Authorship Contributions. Drs. Peterson, Lustberg, and Isakoff conceived of this article, and developed the outline and content. Writing of the content was done by these three authors. Dr. Tolaney, Ms. Ross, and Ms. Salehi reviewed and revised content.

Disclosures. No named authors (Dr. Lustberg, Dr. Peterson, Dr. Tolaney, Ms. Ross, Ms. Salehi, and Dr. Isakoff) have anything to disclose.

Compliance with Ethics Guidelines. This article is based on previously conducted studies and does not contain any studies with human participants or animals performed by any of the authors.

Data Availability. Data sharing is not applicable to this article as no datasets were generated or analyzed during the current study.
Open Access. This article is licensed under a Creative Commons Attribution-NonCommercial 4.0 International License, which permits any non-commercial use, sharing, adaptation, distribution and reproduction in any medium or format, as long as you give appropriate credit to the original author(s) and the source, provide a link to the Creative Commons licence, and indicate if changes were made. The images or other third party material in this article are included in the article's Creative Commons licence, unless indicated otherwise in a credit line to the material. If material is not included in the article's Creative Commons licence and your intended use is not permitted by statutory regulation or exceeds the permitted use, you will need to obtain permission directly from the copyright holder. To view a copy of this licence, visit http://creativecommons.org/licenses/by$\mathrm{nc} / 4.0 /$.

\section{REFERENCES}

1. Komen MMC, Smorenberg CH, van den Hurk CJG, Norier JWR. Factors influencing the effectiveness of scalp cooling in the prevention of chemotherapyinduced alopecia. Oncologist. 2013;18:885-91.

2. Lemieux J, Maunsell E, Provencher L. Chemotherapy-induced alopecia and effects on quality of life among women with breast cancer: a literature review. Psycho Oncol. 2008;17:317-28. https://doi. org/10.1002/pon.1245.

3. Van den Hurk CJ, Mols F, Vingerhoets AJ, Breed WP. Impact of alopecia and scalp cooling on the well-being of breast cancer patients. Psycho Oncol. 2010;19:701-9. https://doi.org/10.1002/pon.1615.

4. American Cancer Society. Cooling caps (scalp hypothermia) to reduce hair loss. https://www. cancer.org/treatment/treatments-and-side-effects/ physical-side-effects/hair-loss/cold-caps.html. Accessed Apr 20, 2020.

5. The ASCO Post. FDA clears expanded indication of scalp-cooling system. June 18, 2018. https://www. ascopost.com/News/58963. Accessed Apr 20, 2020.

6. NCCN Clinical Practice Guidelines in Oncology $\left(\right.$ NCCN Guidelines $^{\circledR}$ ) for Breast Cancer V.3.2020. ( National Comprehensive Cancer Network, Inc. 2019. 
7. NCCN Clinical Practice Guidelines in Oncology (NCCN Guidelines ${ }^{\circledR}$ ) for Ovarian Cancer Including Fallopian Tube Cancer an Primary Peritoneal Cancer V.1.2020. (C) National Comprehensive Cancer Network, Inc. 2019.

8. Rugo HS, Klein P, Melin SA, et al. Association between use of a scalp cooling device and alopecia after chemotherapy for breast cancer. JAMA. 2017;317(6):606-14. https://doi.org/10.1001/jama. 2016.21038 .

9. Nangia J, Wang T, Osborne C, et al. Effect of a scalp cooling device on alopecia in women undergoing chemotherapy for breast cancer. The SCALP Randomized Clinical Trial. JAMA. 2017;317(6):596605. https://doi.org/10.1001/jama.2016.20939.

10. Gianotti E, Razzini G, Bini M, et al. Scalp cooling in daily clinical practice for breast cancer patients undergoing curative chemotherapy: a multicenter interventional study. Asia Pac J Oncol Nurs. 2019;6: 277-82.

11. Bajpai J, Kagwade S, Chandrasekharan A, et al. Randomised controlled trial of scalp cooling for the prevention of chemotherapy induced alopecia. Breast. 2020;40:187-93.

12. Kinoshita T, Nakayama T, Fukuma E, et al. Efficacy of scalp cooling in preventing and recovering from chemotherapy-induced alopecia in breast cancer patients: the HOPE study. Front Oncol 2019; 9. Article 733.

13. Giarratano T, Frezzini $S$, Zaocco $M$, et al. Use of scalp cooling device to prevent alopecia for early breast cancer patients receiving chemotherapy: a prospective study. Breast J. 2019;00:1-6.

14. Kruse M, Abraham J. Management of chemotherapy-induced alopecia with scalp cooling. J Oncol Pract. 2018;14(3):149-54.

15. Betticher DC, Delmore G, Breitenstein U, et al. Efficacy and tolerability of two scalp cooling systems for the prevention of alopecia associated with docetaxel treatment. Support Care Cancer. 2013;21: 2565-73.

16. Van den Hurk CJ, Peerbooms M, van de Poll-Franse LV, Nortier JW, Coebergh JWW, Breed WP. Scalp cooling for hair preservation and associated characteristics in 1411 chemotherapy patients-results of the Dutch Scalp Cooling Registry. Acta Oncol. 2012;00:1-8.

17. Vasconcelos I, Wiesske A, Schoenegg W. Scalp cooling successfully prevents alopecia in breast cancer patients undergoing anthracycline/taxanebased chemotherapy. Breast. 2018;40:1-3.

18. Lemieux J, Provencher L, Perron L, et al. No effect of scalp cooling on survival among women with breast cancer. Breast Cancer Res Treat. 2015;149(1):263-8. https://doi.org/10.1007/s10549-014-3231-0.

19. Rugo HS, Melin SA, Voigt J. Scalp cooling with adjuvant/neoadjuvant chemotherapy for breast cancer and the risk of scalp metastases: systematic review and meta-analysis. Breast Cancer Res Treat. 2017;163:199-205.

20. Paxman data and reference. https://coldcap.com/ data-and-reference. Accessed May 11, 2020.

21. Paxman locations. https://coldcap.com/locations/. Accessed May 11, 2020.

22. Dignitana 2019 year-end report. https://dignitana. com/wp-content/uploads/2020/02/2019yearendrepo rt-1200813-EN.pdf. Accessed June 5, 2020.

23. Komen M, Breed WPM, Smorenburg CH, et al. Results of 20- versus 45-min post-infusion scalp cooling time in the prevention of docetaxel-induced alopecia. Support Care Cancer. 2016;24(6): 2735-42.

24. Saulet D, Riley A. The 2020 HOPPS final rule brings major changes to physician supervision-and more-for cancer programs. https://www.advisory. com/research/oncology-roundtable/oncology-rounds/ 2019/11/hopps-takeaways-cancer-programs. Accessed June 7, 2020.

25. Fischer-Cartlidge E, Ross M, Hernandez K, Featherstone A, Haase C. Scalp cooling. Implementation of a program at a multisite organization. Clin J Oncol Nurs. 2018;22(5):534-41.

26. CMS Benefit Policy Manual. https://www.cms.gov/ Regulations-and-Guidance/Guidance/Manuals/down loads/bp102c15.pdf. Accessed Apr 24, 2020.

27. Elder CT. Oncology supportive care. ACCP updates in therapeutics ${ }^{\circledR}$ 2018: 2-605-645. 